\title{
INFLUÊNCIA DE DIFERENTES DOSES DE TORTA DE FILTRO NO SOLO SOBRE A MIGRAÇÃO E PERSISTÊNCIA DE STEINERNEMA GLASERI (STEINER, 1929) (NEMATODA:RHABDITIDA)
}

\author{
INFLUENCE OF DIFFERENTIAL DOSES OF \\ FILTERCAKE ADDED TO SOIL ON THE MIGRATION \\ AND PERSISTENCE OF STEINERNEMA GLASERI \\ (STEINER, 1929) (NEMATODA:RHABDITIDA)
}

\section{Rodrigues, R.C.D. ${ }^{1}$, Aguillera, M.M. ${ }^{1}$, Gobbi, N. ${ }^{2}$; Pizano, M.A. ${ }^{2}$}

${ }^{1}$ Departamento de Biotecnologia Vegetal, Universidade Federal de São Carlos (UFSCar), Centro de Ciências Agrárias (CCA), Caixa Postal:153, CEP 13600970,Araras, SP E-Mail: regina@dbv.cca.ufscar.br, marineide@dbv.cca.ufscar.br, ${ }^{2}$ Departamento de Ecologia, Universidade Estadual Paulista, Instituto de Biociências, Caixa Postal: 199, CEP 13506-900, Rio Claro, SP E-mail: ngobbi@rc.unesp.br, mapizano@rc.unesp.br

\section{RESUMO}

Nematóides entomopatogênicos são usados no controle biológico de vários insetos/praga. Ocorrem naturalmente, e há grande interesse em conhecer as condições do ambiente que possam favorecer suas atividades no solo para localizar hospedeiros potenciais e sua sobrevivência. O objetivo deste trabalho foi determinar o efeito da torta de filtro (T.F.) adicionada ao solo (SO) sobre a migração e persistência de Steinernema glaseri. Os tratamentos consistiram de 0, 25, 50, 75 e 100\% de T.F. adicionados em solo esterilizado: arenoso e argiloso. Galleria mellonella (Lepidoptera:Pyralidae) foi usada como hospedeiro indicador para a atividade dos juvenis infectivos do nematóide. No solo arenoso, a adição de T.F. em proporção de até $50 \%$ não influenciou a migração de nematóides em direção ao hospedeiro, enquanto em solo argiloso a proporção máxima onde ocorreu a migração foi de $25 \%$ T.F. Os juvenis infectivos moveram-se mais para a superfície do que para o fundo da coluna quando o substrato continha solo arenoso. Em solo argiloso, a movimentação foi semelhante nas duas direções em todos os teores de T.F. A persistência do nematóide foi mais elevada em substratos com solo argiloso, nos tratamentos com maior proporção de solo em relação à torta de filtro. 
Palavras-chave: nematóides entomopatogênicos, matéria orgânica, migração, persistência, Steinernema glaseri.

\begin{abstract}
Entomopathogenic nematodes are commonly used for the biological control of many insects/pests. Besides their natural occurrence, there is a great interest in the study of environmental conditions, which can favor their activities in the soil in order to find potential hosts and survivors. The objective of this research was to determine the effects of filtercake added to soil on migration and persistence of Steinernema glaseri. The treatments were: $0 ; 25 ; 50 ; 75$; and $100 \%$ filtercake added to two types of sterilized soil: sandy and clay. Galleria mellonella (Lepidoptera: Pyralidae) was used as an indicator host for the nematode activity. In the sandy soil, additions of up to $50 \%$ filtercake have no influence on nematode migration towards the host, while in clay soil the maximum proportion in which migration occurred with $25 \%$ filtercake. Infective juveniles moved more to the top than to the bottom column, in sandy soil. In clay soil the migration was similar in both directions at all filtercake levels. The nematode persistence was higher in clay soil substrates, in the treatments with higher proportion of soil in relation to filtercake.
\end{abstract}

Key words: entomopathogenic nematodes, organic matter, migration, persistence, Steinernema glaseri.

\title{
1. INTRODUÇÃO
}

Nematóides entomopatogênicos do gênero Steinernema Travassos, 1927 (Rhabditida: Steinernematidae) são importantes agentes de controle biológico de insetos e têm mostrado, através de experimentos de laboratório e casas de vegetação, grande habilidade em localizar, invadir o corpo e matar hospedeiros pertencentes a diversas ordens (POINAR, 1979). Podem ser produzidos em larga escala tanto "in vivo" como "in vitro" e não apresentam os inconvenientes dos produtos químicos (GAUGLER, 1988). Em diversas pragas de importância econômica no Brasil, apresentam-se como candidatos potenciais para o controle biológico. De fato, desde 1985, nematóides do gênero Steinernema têm sido isolados de solos no estado de São Paulo (PIZANO et al.,1985).

Embora testes de patogenicidade tenham mostrado a suscetibilidade de hospedeiros, tais como Migdolus fryanus (Coleoptera: Cerambycidae), o nível de incidência desta praga é alto em local de ocorrência do nematóide. Torna-se necessário, portanto, estudar os efeitos de medidas que possam interferir nas 
atividades dos nematóides, visando o manejo adequado para propiciar o aumento de populações nativas, bem como o estabelecimento de nematóides introduzidos em novos locais.

Sabe-se que os teores de umidade e de matéria orgânica no solo podem limitar a atividade desses nematóides (KAYA, 1990). A adição de compostos orgânicos e inorgânicos para melhorar a fertilidade do solo e o crescimento das plantas está entre as práticas freqüentemente usadas na agricultura, e muitos destes melhoramentos podem interferir no desenvolvimento de nematóides benéficos no solo (MULLER e GOOCH, 1982; RODRIGUEZ-KÁBANA, 1986; BEDNAREK e GAUGLER, 1997).

A torta de filtro é um resíduo proveniente da filtração a vácuo da mistura de lodo dos decantadores com bagacinho, no processo de produção de açúcar. O resíduo é um material rico em matéria orgânica, cálcio, fósforo e nitrogênio; dos micronutrientes, o ferro aparece com destaque. Este subproduto é largamente utilizado em áreas cultivadas com cana de açúcar, principalmente em solos arenosos, onde, coincidentemente, as pragas de solo são mais prejudiciais (ORLANDO FILHO et al., 1983).

Desta forma, considerando a hipótese de a matéria orgânica se constituir em importante fator para a migração e sobrevivência de nematóides entomopatogênicos, objetivou-se neste trabalho estudar os efeitos da torta de filtro adicionada ao solo sobre a dispersão e persistência de Steinernema glaseri.

\section{MATERIAL E MÉTODOS}

Os experimentos foram conduzidos em condições de laboratório, no Departamento de Biotecnologia Vegetal, do Centro de Ciências Agrárias da Universidade Federal de São Carlos, utilizando-se o nematóide entomopatogênico Steinernema glaseri strain Sta Rosa. Este foi multiplicado em lagartas de $7^{\circ}$ instar (estádio larval) (ORLANDO FILHO et al, 1983) de Galleria mellonella L. (Lepidoptera, Pyralidae), de acordo com metodologia desenvolvida por Dutky et al. (1964). Juvenis infectivos foram coletados em armadilha de White (POINAR, 1979) (constituída por placa de petri com $15 \mathrm{~cm}$ de diâmetro contendo uma tampa invertida de placa de petri de $9 \mathrm{~cm}$ de diâmetro, e sobre esta, papel filtro de $12 \mathrm{~cm}$ de diâmetro moldado de forma que os bordos fiquem em contato com água destilada colocada na placa maior) e, posteriormente, mantidos na forma de suspensão, à temperatura de aproximadamente $4^{\circ} \mathrm{C}$.

Os experimentos foram conduzidos em dois tipos de solo, um arenoso e outro argiloso, cada um contendo cinco tratamentos, representando diferentes combinações solo/torta de filtro. As análises granulométricas dos solos bem como as análises químicas da torta de filtro foram realizadas no laboratório de Análises Físicas do Departamento de Recursos Naturais e Proteção Ambiental, do Centro de Ciências Agrárias. 
Foram conduzidos dois experimentos: o primeiro para o estudo da migração de $S$. glaseri o segundo para o estudo da persistência deste mesmo nematóide.

\subsection{Migração de Steinernema glaseri}

Este experimento foi conduzido em recipientes plásticos de $17,5 \mathrm{~cm}$ de altura e 9,5 cm de diâmetro, com temperatura $28 \pm 5^{\circ} \mathrm{C}$ e luz ambiente, com os seguintes tratamentos: Tratamento A $-100 \%$ Torta de Filtro (TF), Tratamento B - 75\% TF + $25 \%$ solo (SO), Tratamento C $-50 \% \mathrm{TF}+50 \% \mathrm{SO}$, Tratamento D $-25 \% \mathrm{TF}+75 \%$ SO, Tratamento E - 100\% SO. O delineamento estatístico foi inteiramente ao acaso com 5 tratamentos e 6 repetições, com 6 lagartas de G. mellonella por repetição. Os substratos (torta + solo) foram colocados até completar $14 \mathrm{~cm}$ de altura nos referidos recipientes.

No fundo e na superfície da coluna do substrato foram acondicionadas três lagartas de G. mellonella. No centro da coluna, ou seja, na altura de $7 \mathrm{~cm}$, foi pipetado o inóculo que constou de 10.000 juvenis infectivos (J3) de S. glaseri, contidos em 5,0 mL de suspensão aquosa para assegurar alto nível de infestação no solo.

O nível inicial de umidade foi ajustado, para melhor sobrevivência dos nematóides, em cada tratamento de acordo com avaliação preliminar feita em laboratório ( $24 \%$ para o solo e $12 \%$ para a torta de filtro), pelo método gravimétrico (KRAMER, 1969).

Para determinar a migração de nematóides foram utilizados dois parâmetros: número de lagartas infectadas e número de adultos de $1^{\text {a }}$ geração desenvolvidos no interior destas lagartas (EPSKY e CAPINERA, 1993). Este número representa a quantidade de juvenis infectivos que migraram em direção ao hospedeiro, o invadiram e se tornaram adultos. $\mathrm{O}$ número de lagartas infectadas foi determinado coletando-às 96 horas após terem sido expostas ao ataque dos nematóides e dissecando-as, em seguida, para determinar o número de adultos desenvolvidos.

\subsection{Persistência de Steinernema glaseri}

Foram utilizados os mesmos recipientes e substratos já inoculados para determinação da migração. No solo arenoso foram feitas avaliações aos $7 ; 10 ; 13 ; 16$; $26 ; 36 ; 46 ; 56 ; 66 ; 76$ dias após a inoculação. No solo argiloso as avaliações foram feitas de 3 em 3 dias, começando aos 7 e terminando em 79 dias após a inoculação. Foram colocadas três lagartas de G. mellonella por repetição distribuídas no fundo, no centro e na superfície da coluna. Assim, as lagartas permaneciam 72 horas expostas em cada época de avaliação e eram submetidas às mesmas observações do experimento anterior.

A determinação de maior período após as primeiras avaliações no solo arenoso foi devida a significativa redução no número de nematóides adultos nas lagartas.

A sobrevivência dos nematóides foi determinada pela habilidade dos juvenis infectivos de alcançar, infectar e se desenvolver em adultos nas lagartas de $G$. 
mellonella colocadas na superfície, no meio e no fundo dos recipientes contendo os substratos. O delineamento estatístico foi inteiramente ao acaso com 5 tratamentos, 6 repetições e 3 lagartas por repetição.

Em ambos os experimentos, a análise estatística dos dados foi feita utilizandose o Software SAS - Statistical Analysis System, versão 6.11, e as médias comparadas pelo Teste de Tukey a $5 \%$ de probabilidade. Os dados foram transformados método arcsen da $\sqrt{\frac{\%}{100} \text {. }}$.

\section{RESULTADOS E DISCUSSÃO}

\subsection{Migração de Steinernema glaseri}

Nos substratos com solo arenoso, houve diferença significativa entre os tratamentos quanto ao número de lagartas infectadas, sendo que o tratamento contendo apenas solo apresentou o maior número e os tratamentos apenas com torta de filtro e com $75 \%$ deste material mostraram os menores valores em relação a este parâmetro (Tabela 1). A porcentagem de lagartas infectadas pelo nematóide variou de 61 a $100 \%$ na superfície e, de 50 a $94 \%$ no fundo da coluna, sendo geralmente mais alta nos substratos onde a proporção de solo era igual ou mais elevada que a torta de filtro.

Estes dados demonstram que os nematóides tendem a migrar mais intensamente em substratos com menores teores de torta de filtro, em ambas as direções, superfície e fundo da coluna. Quanto ao direcionamento dos nematóides para a superfície ou para o fundo da coluna, observou-se que não houve diferença significativa, ao nível de $5 \%$ de probabilidade.

No solo arenoso, o número de nematóides adultos encontrados dentro das lagartas de G. mellonella variou significativamente e seguiu a mesma tendência indicada pelo número de lagartas infectadas, ou seja, foi maior nos substratos com menor teor de torta de filtro. No tratamento constituído apenas por torta de filtro, bem como naquele que recebeu $75 \%$ deste material observou-se o menor número de adultos, enquanto que no tratamento onde foi utilizado o solo nas proporções de 100, 75 e $50 \%$, o número de nematóides adultos por lagarta foi significativamente maior (Tabela 2).

Independentemente do substrato (torta /solo), foi obtido número significativamente mais elevado de nematóides na superfície da coluna em relação ao fundo (Tabela 2). Este comportamento está em concordância com o que foi observado por (SCHROEDER e BEAVERS, 1987), os quais verificaram que $S$. glaseri, diferentemente de outras espécies de Steinernema e Heterorhabditis, migra mais em direção à superfície.

Nos substratos com solo argiloso o número de lagartas infectadas não variou entre os tratamentos. Quanto ao parasitismo das lagartas na superfície ou fundo da coluna, independentemente dos tratamentos, houve diferença significativa, entre as 
médias das duas posições, indicando maior migração dos nematóides para a superfície (Tabela 3).

Da mesma forma que o número de lagartas infectadas, a variação no número médio de nematóides que infectaram as lagartas no solo argiloso não foi significativa, entre os diferentes tratamentos e posições na coluna de substrato (Tabela 4).

Independentemente dos tratamentos utilizados, comparando-se os dados médios totais do número de nematóides nas lagartas infectadas, neste solo, com os dados obtidos em solo arenoso, observou-se neste último maior movimentação, em ambas as direções (Figura1).

Esta observação está de acordo com o que é conhecido sobre movimentação de nematóides em relação a tamanho de partículas de solo. A porosidade afeta a movimentação dos nematóides e, desta forma, ocorre menor migração com o aumento da porcentagem de silte e argila no solo. $\mathrm{O}$ nematóide não pode mover-se entre as partículas de solo quando o diâmetro for menor do que o comprimento do nematóide (GEORGIS e POINAR, 1983). Juvenis infectivos de S. glaseri, utilizados nesta pesquisa, medem de 864 a $1448 \mu \mathrm{m}$. Poinar (1986) considera que estes nematóides estão classificados entre os maiores do gênero Steinernema. Desta forma, a sua movimentação é favorecida em solo arenoso, o qual possui partículas maiores $(0,02$ a $2 \mathrm{~mm})$ do que solo argiloso $(<0,002 \mathrm{~mm})$. Kaya (1990) também considera que a textura do solo afeta a habilidade dos nematóides de infectarem o hospedeiro, bem como sua habilidade de dispersão vertical.

\subsection{Persistência de Steinernema glaseri}

Analisando os efeitos da torta de filtro sobre a persistência de $S$. glaseri, em substratos com solo arenoso, a análise estatística realizada com o número de lagartas infectadas, apresentada na Tabela 5, mostrou que não houve diferenças significativas. Mesmo assim, continuou-se observando as lagartas no período de 76 dias. No entanto, no final deste período houve recuperação de lagartas infectadas nos tratamentos com 100 e $75 \%$ de $\mathrm{TF}$, demonstrando assim sua persistência até este período. Apesar de não haver diferença estatística, observou-se maior quantidade de lagartas infectadas pelo nematóide no tratamento $100 \%$ solo.

Para o número médio de nematóides adultos de $S$.glaseri em G. mellonella neste solo, também foi feita análise estatística até os 16 primeiros dias como observase na Tabela 6.

A persistência dos nematóides foi afetada pelo tipo de substrato e a maior persistência ocorreu quando a quantidade de solo arenoso era maior do que a de torta de filtro (TF). Avaliações feitas aos 66 e 76 dias, mostraram que havia nematóides vivos, embora em pequenas quantidades.

Quando foi utilizado o solo argiloso, observou-se que o número de lagartas infectadas foi maior onde a proporção de solo era maior e persistiu até o final das avaliações com 79 dias, apesar da análise estatística ter sido feita até os 16 dias, como no solo arenoso (Tabela 7). 
Para o número de nematóides, a persistência foi significativamente mais elevada nos tratamentos com $50 \%$ ou mais de solo no substrato. Quando comparado com solo arenoso o número de nematóides foi menor (Tabela 8 ).

\section{CONCLUSÕES}

O nematóide entomopatogênico $S$. glaseri apresenta a habilidade de migrar verticalmente e infectar lagartas de G. mellonella em substratos com diferentes teores de torta de filtro, mesmo naqueles com alto teor $(100 \%)$ deste composto.

A textura do solo influencia a movimentação dos nematóides, sendo esta movimentação mais intensa em solo arenoso do que em solo argiloso.

Em solo arenoso, a migração é maior em direção à superfície do que em direção ao fundo da coluna. Já em solo argiloso, a migração ocorre igualmente nos dois sentidos.

Os nematóides podem persistir por um período de 76 dias em solo arenoso e 79 dias em solo argiloso.

As proporções de até 25 e $50 \%$ de torta de filtro em solo arenoso e de até $25 \%$ em solo argiloso, não apresentam efeitos negativos à S. glaseri. Acima dessas proporções a torta de filtro atuou de forma negativa do desenvolvimento do nematóide.

\section{AGRADECIMENTOS}

Ao Prof. Dr. Norberto Antonio Lavorenti, do Departamento de Tecnologia Agroindustrial e Sócio Economia Rural da Universidade Federal de São Carlos campus de Araras (CCA), pela orientação quanto às análises estatísticas e valiosas sugestões.

Às Biólogas Regina Helena de Carvalho Assumpção e Lauricema Barbosa Lozada Marchetti, do Departamento de Biotecnologia Vegetal da UFSCar, campus de Araras (CCA), pela amizade, incentivo e colaboração na parte prática do trabalho.

\section{REFERÊNCIAS}

BEDNAREK, A.; GAUGLER, R. Compatibility of soil amendments with entomopathogenic nematodes. Journal of Nematology, v. 2, n. 29, p. 220-227, 1997.

DUTKY, S.R.; THOMPSON, J.V.; CANTWELL, G.E. A technique for the mass propagation of the DD-136 nematode. Journal Insect Pathology, n.6, p. 417-422, 1964. 
EPSKY, N.D.; CAPINERA, J.L. Quantification of invasion of two strains of Steinernema carpocapsae (Weiser) into three lepidopteran larvae. Journal of Nematology, v.2, n. 25, p. 173-180, 1993.

GAUGLER, R. Ecological consideration in the biological control of soil inhabiting insects with entomopathogenic nematodes. Agriculture Ecosystem Environmental, n.24, p. 351-360, 1988.

GEORGIS, R.; POINAR JR., G.O. Effect of soil texture on the distribution and infectivity of Neoaplectana glaseri (Nematoda: Steinernematidae). Journal of Nematology, n.15, p. 329-332, 1983.

KAYA, H.K. Soil ecology. In: GLAUGLER, R., KAYA, H.K. (eds.). Entomopathogenic nematodes in biological control. Boca Raton: CRC Press, 1990. p. 93-115.

KRAMER, P.J. Plant and soil water relationships: a modern synthesis. New York, Mcgraw-Hill, 1969. 482p.

MULLER, R.; GOOCH, P.S. Organic amendments in nematode control. An examination of the literature. Nematropica, n. 12, p. 319-326, 1982.

ORLANDO FILHO, J.; SILVA, G. M.A.; LEME, E.J.A. Utilização agrícola dos resíduos da agroindústria canavieira. In: ORLANDO FILHO, J. (Coord.). Nutrição e adubação da cana-de-açúcar no Brasil. Piracicaba: Instituto do Açúcar e do Álcool, 1983. p.229-264.

PIZANO, M.A.; AGUILLERA, M.M.; MONTEIRO, A.R.; FERRAZ, L.C.C.B. Incidência de Neoaplectana glaseri parasitando ovo de Migdolus fryanus (Coleoptera: Cerambycidae). Nematologia Brasileira, n. 9, p. 9-10, 1985.

POINAR, JR., G.O. Nematodes for biological control of insects. Boca Raton: CRC Press, 1979. $277 \mathrm{p}$.

POINAR, JR., G.O. Recognition of the Neoaplectana species. Proceedings of the Helminthological Society of Washington, v. 1, n. 53, p. 121-129, 1986.

RODRIGUEZ-KABANA, R. Organic and inorganic nitrogen amendments to soil as nematode suppressants. Journal of Nematology, n. 18, p. 129-135, 1986.

SCHROEDER, W.J.; BEAVERS, J.B. Movement of the entomogenous nematodes of the families Heterorhabditidae and Steinernematidae in soil. Journal of Nematology, Flórida, v. 2, n. 19, p. 257-259, 1987. 
Tabela 1. Número total e porcentagem de lagartas de Galleria mellonella infectadas por Steinernema glaseri, em substratos compostos por solo (SO) arenoso e/ou torta de filtro (TF) após migração para a superfície ou para o fundo da coluna.

\begin{tabular}{|c|c|c|c|c|c|c|c|}
\hline \multirow{2}{*}{ Tratamentos } & \multicolumn{2}{|c|}{ Superf. da coluna } & \multicolumn{2}{|c|}{ Fundo da coluna } & \multicolumn{2}{|c|}{ Total } & \multirow[t]{2}{*}{$\begin{array}{c}\text { Médias } \\
\text { transform.* }\end{array}$} \\
\hline & $\mathrm{N}^{\mathrm{o}}$ & $\%$ & $\mathrm{~N}^{\mathrm{o}}$ & $\%$ & $\mathrm{~N}^{\mathrm{o}}$ & $\%$ & \\
\hline $100 \% \mathrm{TF}$ & 14 & 77,78 & 9 & 50,0 & 23 & 63,89 & $1,92 b$ \\
\hline $75 \% \mathrm{TF}+25 \% \mathrm{SO}$ & 11 & 61,11 & 11 & 61,11 & 22 & 61,11 & $1,83 b$ \\
\hline $50 \% \mathrm{TF}+50 \% \mathrm{SO}$ & 14 & 77,78 & 17 & 94,44 & 31 & 86,11 & $2,58 \mathrm{ab}$ \\
\hline $25 \% \mathrm{TF}+75 \% \mathrm{SO}$ & 16 & 88,89 & 15 & 83,33 & 31 & 86,11 & $2,58 \mathrm{ab}$ \\
\hline $100 \% \mathrm{SO}$ & 18 & 100,0 & 17 & 94,44 & 35 & 97,22 & $2,92 \mathrm{a}$ \\
\hline Médias transform.* & $2,43 \mathrm{a}$ & & $2,30 \mathrm{a}$ & & & & \\
\hline
\end{tabular}

*Médias seguidas de letras iguais não diferem estatisticamente, ao nível de 5\% de probabilidade, pelo teste de Tukey.

Tabela 2. Números médios de nematóides adultos de Steinernema glaseri em lagartas de Galleria mellonella em duas diferentes posições na coluna de substrato, em solo (SO) arenoso.

\begin{tabular}{|l|c|c|c|c|}
\hline Tratamentos & $\begin{array}{c}\text { Superfície da } \\
\text { coluna }\end{array}$ & Fundo da coluna & Médias originais & $\begin{array}{c}\text { Médias } \\
\text { transform.* }\end{array}$ \\
\hline $100 \% \mathrm{TF}$ & 36,17 & 61,67 & 48,92 & $3,09 \mathrm{c}$ \\
\hline $75 \% \mathrm{TF}+25 \% \mathrm{SO}$ & 92,67 & 62,83 & 77,75 & $3,47 \mathrm{bc}$ \\
\hline $50 \% \mathrm{TF}+50 \% \mathrm{SO}$ & 328,0 & 63,50 & 195,75 & $4,77 \mathrm{ab}$ \\
\hline $25 \% \mathrm{TF}+75 \% \mathrm{SO}$ & 354,67 & 73,17 & 213,92 & $4,92 \mathrm{ab}$ \\
\hline $100 \% \mathrm{SO}$ & 361,83 & 244,17 & 303,00 & $5,55 \mathrm{a}$ \\
\hline Médias transform.* & $4,72 \mathrm{a}$ & $3,99 \mathrm{~b}$ & & \\
\hline
\end{tabular}

* Médias seguidas de letras iguais não diferem estatisticamente, ao nível de 5\% de probabilidade, pelo teste de Tukey. 
Tabela 3. Número e porcentagem de lagartas de Galleria mellonella infectadas por Steinernema glaseri, em substratos compostos por solo (SO) argiloso e/ou torta de filtro (TF) após migração para a superfície ou para o fundo da coluna.

\begin{tabular}{|c|c|c|c|c|c|c|c|}
\hline \multirow[t]{2}{*}{ Tratamentos } & \multicolumn{2}{|c|}{$\begin{array}{l}\text { Superfície } \\
\text { da coluna }\end{array}$} & \multicolumn{2}{|c|}{ Fundo da coluna } & \multicolumn{2}{|c|}{ Total } & \multirow{2}{*}{$\begin{array}{c}\text { Médias } \\
\text { Transform.* }\end{array}$} \\
\hline & $\mathrm{N}^{\mathrm{o}}$ & $\%$ & $\mathrm{~N}^{\mathrm{o}}$ & $\%$ & $\mathrm{~N}^{\mathrm{o}}$ & $\%$ & \\
\hline $100 \% \mathrm{TF}$ & 17 & 94,44 & 18 & 100,00 & 35 & 97,22 & $2,92 \mathrm{a}$ \\
\hline $75 \% \mathrm{TF}+25 \% \mathrm{SO}$ & 13 & 72,22 & 12 & 66,67 & 25 & 69,44 & $2,08 \mathrm{a}$ \\
\hline $50 \% \mathrm{TF}+50 \% \mathrm{SO}$ & 15 & 83,33 & 13 & 72,22 & 28 & 77,78 & $2,33 \mathrm{a}$ \\
\hline $25 \% \mathrm{TF}+75 \% \mathrm{SO}$ & 15 & 83,33 & 11 & 61,11 & 26 & 72,22 & $2,17 \mathrm{a}$ \\
\hline $100 \% \mathrm{SO}$ & 16 & 88,89 & 12 & 66,67 & 28 & 77,78 & $2,33 \mathrm{a}$ \\
\hline Médias transform.* & $2,53 \mathrm{a}$ & & $2,20 \mathrm{~b}$ & & & & \\
\hline
\end{tabular}

* Médias seguidas por letras iguais não diferem entre si, ao nível de 5\% de probabilidade, pelo teste de Tukey.

Tabela 4. Números médios de nematóides adultos de Steinernema glaseri em lagartas de Galleria mellonella em duas diferentes posições na coluna de substrato, em solo (SO) argiloso.

\begin{tabular}{|l|c|c|c|c|}
\hline Tratamentos & $\begin{array}{c}\text { Superfície da } \\
\text { coluna }\end{array}$ & Fundo da coluna & Médias & Médias \\
originais & transform.* \\
\hline $100 \% \mathrm{TF}$ & 14,17 & 14,17 & 14,17 & $2,34 \mathrm{a}$ \\
\hline $75 \% \mathrm{TF}+25 \% \mathrm{SO}$ & 11,00 & 16,17 & 13,59 & $2,18 \mathrm{a}$ \\
\hline $25 \% \mathrm{TF}+75 \% \mathrm{SO}$ & 18,00 & 17,00 & 17,50 & $2,56 \mathrm{a}$ \\
\hline $100 \% \mathrm{SO}+50 \% \mathrm{SO}$ & 56,17 & 31,17 & 43,67 & $3,22 \mathrm{a}$ \\
\hline Médias transform.* & $2,95 \mathrm{a}$ & $2,56 \mathrm{a}$ & & $3,47 \mathrm{a}$ \\
\hline
\end{tabular}

*Médias seguidas por letras iguais que não diferem entre si, ao nível de 5\% de probabilidade, pelo teste de Tukey 
Tabela 5. Médias originais dos efeitos principais e da interação, para lagartas infectadas em experimento de persistência, para o solo (SO) arenoso.

\begin{tabular}{|c|c|c|c|c|c|}
\hline \multirow{2}{*}{ Tratamentos } & \multicolumn{4}{|c|}{ Épocas/Dias } & \multirow{2}{*}{ Médias de Tratamentos* } \\
\hline & 7 & 10 & 13 & 16 & \\
\hline $100 \% \mathrm{TF}$ & 1,51 & 1,50 & 1,51 & 1,40 & $1,48 \mathrm{a}$ \\
\hline $75 \% \mathrm{TF}+25 \% \mathrm{SO}$ & 1,45 & 1,38 & 1,57 & 1,35 & $1,44 \mathrm{a}$ \\
\hline $50 \% \mathrm{TF}+50 \% \mathrm{SO}$ & 1,57 & 1,35 & 1,38 & 1,25 & $1,39 \mathrm{a}$ \\
\hline $25 \% \mathrm{TF}+75 \% \mathrm{SO}$ & 1,57 & 1,51 & 1,42 & 1,36 & $1,47 \mathrm{a}$ \\
\hline $100 \% \mathrm{SO}$ & 1,77 & 1,61 & 1,53 & 1,52 & $1,61 \mathrm{a}$ \\
\hline Médias Épocas* & $1,57 \mathrm{a}$ & $1,47 \mathrm{a}$ & $1,48^{\mathrm{a}}$ & $1,38 \mathrm{a}$ & \\
\hline
\end{tabular}

* Médias seguidas por letras iguais não diferem estatisticamente, ao nível de 5\% de probabilidade, pelo teste de Tukey.

Tabela 6. Médias transformadas dos efeitos principais e da interação para o número de nematóides, em experimento de persistência, para o solo (SO) arenoso.

\begin{tabular}{|l|c|c|c|c|c|}
\hline \multirow{2}{*}{ Tratamentos } & \multicolumn{4}{|c|}{ Épocas/Dias } & \multirow{2}{*}{ Médias de } \\
\cline { 2 - 5 } & 7 & 10 & 13 & 16 & Tratamentos* \\
\hline $100 \% \mathrm{TF}$ & 3,93 & 2,38 & 2,93 & 1,82 & $2,77 \mathrm{ab}$ \\
\hline $75 \% \mathrm{TF}+25 \% \mathrm{SO}$ & 3,35 & 1,81 & 2,71 & 1,36 & $2,31 \mathrm{~b}$ \\
\hline $50 \% \mathrm{TF}+50 \% \mathrm{SO}$ & 3,29 & 2,40 & 1,88 & 1,43 & $2,25 \mathrm{~b}$ \\
\hline $25 \% \mathrm{TF}+75 \% \mathrm{SO}$ & 3,42 & 2,86 & 2,24 & 1,44 & $2,49 \mathrm{ab}$ \\
\hline $100 \% \mathrm{SO}$ & 4,74 & 2,96 & 3,27 & 2,85 & $3,46^{\mathrm{a}}$ \\
\hline Médias Épocas* & $3,74 \mathrm{a}$ & $2,48 \mathrm{~b}$ & $2,61 \mathrm{~b}$ & $1,78 \mathrm{c}$ & \\
\hline
\end{tabular}

*Médias seguidas por letras iguais não diferem estatisticamente, ao nível de 5\%de probabilidade, pelo teste de Tukey. 
Tabela 7. Médias transformadas dos efeitos principais e da interação, para o número de lagartas infectadas em experimento de persistência, para o solo (SO) argiloso.

\begin{tabular}{|c|c|c|c|c|c|}
\hline \multirow{2}{*}{ Tratamentos } & \multicolumn{4}{|c|}{ Épocas/Dias } & Médias dos \\
\cline { 2 - 5 } & 7 & 10 & 13 & 16 & Tratamentos* \\
\hline $100 \% \mathrm{TF}$ & 1,08 & 0,96 & 0,79 & 0,71 & $0,89 \mathrm{c}$ \\
\hline $75 \% \mathrm{TF}+25 \% \mathrm{SO}$ & 1,40 & 1,45 & 0,88 & 0,96 & $1,17 \mathrm{~b}$ \\
\hline $50 \% \mathrm{TF}+50 \% \mathrm{SO}$ & 1,48 & 1,31 & 1,57 & 0,94 & $1,32 \mathrm{ab}$ \\
\hline $25 \% \mathrm{TF}+75 \% \mathrm{SO}$ & 1,72 & 1,28 & 1,62 & 1,20 & $1,46 \mathrm{a}$ \\
\hline $100 \% \mathrm{SO}$ & 1,45 & 1,42 & 1,67 & 1,38 & $1,48 \mathrm{a}$ \\
\hline Médias Épocas* & $1,43 \mathrm{a}$ & $1,28 \mathrm{a}$ & $1,31 \mathrm{a}$ & $1,04 \mathrm{~b}$ & \\
\hline
\end{tabular}

* Médias seguidas por letras iguais não diferem estatisticamente, ao nível de 5\% de probabilidade, pelo teste de Tukey.

Tabela 8. Médias transformadas dos efeitos principais e da interação, para o número de nematóides em experimento de persistência, para o solo(SO) argiloso.

\begin{tabular}{|c|c|c|c|c|c|}
\hline \multirow{2}{*}{ Tratamentos } & \multicolumn{4}{|c|}{ Épocas/Dias } & \multirow{2}{*}{ Médias dos } \\
\cline { 2 - 5 } & 7 & 10 & 13 & 16 & tratamentos* \\
\hline $100 \% \mathrm{TF}$ & 1,01 & 0,71 & 0,80 & 0,00 & $0,63 \mathrm{c}$ \\
\hline $75 \% \mathrm{TF}+25 \% \mathrm{SO}$ & 2,19 & 1,78 & 0,23 & 1,05 & $1,31 \mathrm{bc}$ \\
\hline $50 \% \mathrm{TF}+50 \% \mathrm{SO}$ & 2,86 & 1,91 & 2,91 & 0,48 & $2,04 \mathrm{ab}$ \\
\hline $25 \% \mathrm{TF}+75 \% \mathrm{SO}$ & 3,54 & 1,76 & 2,42 & 1,82 & $2,39 \mathrm{a}$ \\
\hline $100 \% \mathrm{SO}$ & 3,14 & 1,95 & 3,07 & 1,79 & $2,49 \mathrm{a}$ \\
\hline Médias Épocas* & $2,55 \mathrm{a}$ & $1,62 \mathrm{~b}$ & $1,89 \mathrm{ab}$ & $1,03 \mathrm{c}$ & \\
\hline
\end{tabular}

* Médias seguidas por letras iguais não diferem estatisticamente, ao nível de 5\% de probabilidade, pelo teste de Tukey. 


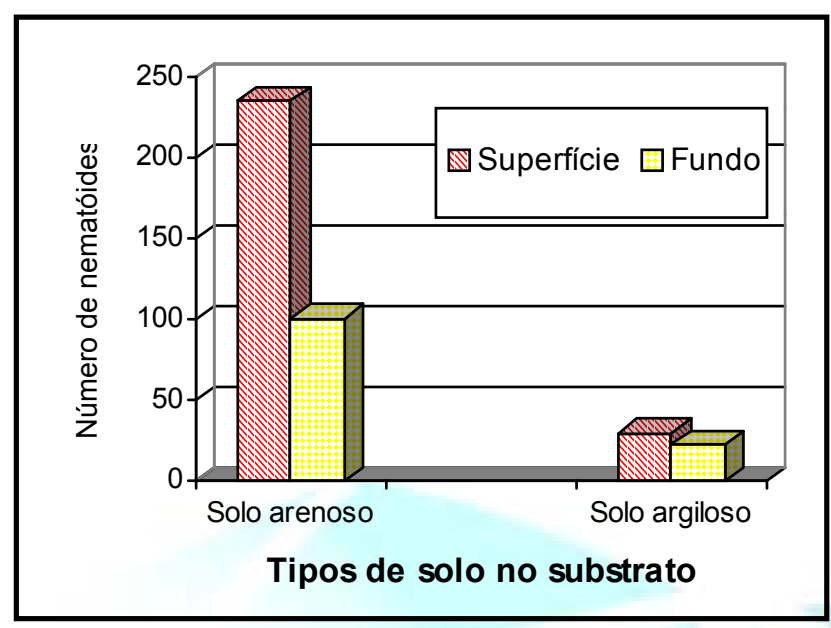

Figura 1. Número de nematóides adultos de S. glaseri em lagartas de G. mellonella em diferentes posições nas colunas de solo arenoso e argiloso. 